\title{
三氟甲基酰腙的氰基化反应研究
}

$\begin{array}{cccc}\text { 徐炜刚 黄丹凤 } & \text { 王克虎 } & \text { 赵芳霞 } & \text { 赵转霞 } \\ & \begin{array}{l}\text { 虎永琴 } \\ \text { (苏灜鹏 }\end{array} & \text { 胡雨来* } & \\ \text { (西北师范大学化学化工学院 } & \text { 兰州 730070) }\end{array}$

\begin{abstract}
摘要 探索了在路易斯酸催化下，三氟甲基酰腙与三甲基氰硅烷的 1,2-加成反应，得到了一系列含有三氟甲基的氰基 酰朋类化合物. 该方法具有反应条件温和、操作简单、产率高等特点，为合成结构多样的含三氟甲基的酰胇类化合物 提供了一种有效的方法.

关键词 三氟甲基酰腙; 三甲基氰硅烷; 酰貄; 氰基化反应
\end{abstract}

\section{Study on the Hydrocyanation Reaction of Trifluoromethylated Acylhydrazones}

\author{
$\mathrm{Xu}$, Weigang \\ Huang, Danfeng \\ Wang, Kehu \\ Zhao, Fangxia \\ Zhao, Zhuanxia \\ $\mathrm{Hu}$, Yongqing \\ $\mathrm{Su}$, Yingpeng \\ $\mathrm{Hu}$, Yulai* \\ (College of Chemistry and Chemical Engineering, Northwest Normal University, Lanzhou 730070)
}

\begin{abstract}
The Lewis acid catalyzed 1,2-addition reaction of trifluoromethylated acylhydrazones with trimethylsilyl cyanide was investigated, and a series of cyanohydrazide compounds containing trifluoromethyl group were afforded in high yield. The method could be easily operated in mild reaction conditions, which provides an efficient method for the synthesis of trifluoromethylated hydrazides with structural diversity.
\end{abstract}

Keywords trifluoromethylated acylhydrazones; trimethylsilyl cyanide; acylhydrazides; hydrocyanation

酰肼及其衍生物是一类重要的有机化合物, 它们不 仅是有机合成中制备含氮化合物常用的合成砌块之 - [1], 而且也是合成染料及液晶材料的重要前体或中间 体 ${ }^{[2]}$. 酰肼类化合物还具有非常重要的生物活性. 早在 1988 年, $N$-叔丁基- $N, N^{\prime}$-双酰肼 RH- $5849^{[3]}(\mathbf{A})$ 就被发现 具有很好的杀虫活性. 随后, 该双酰肼化合物的一系列 衍生物 $(\mathbf{B} \sim \mathbf{E})^{[4]}$ 也被陆续开发出来并被商品化, 从而在 农药领域被广泛用作杀虫剂(图 1). 此外, 一些含有酰肼 结构单元的化合物 $(\mathbf{F} \sim \mathbf{H})$ 因为具有杀菌、消炎和抗肿瘤 等活性 ${ }^{[5]}$, 可作为医药领域中的潜在候选药物. 特别重 要的是, 含有三氟甲基的酰肼化合物(I)已在 2009 年被 报道具有抗癌作用(图 1) ${ }^{[6]}$. 因此, 探索结构新颖的酰肼 类化合物的合成具有重要的意义. 有关酰肼类化合物的 合成, 传统上是通过肼与羧酸或其衍生物, 例如酰氯、 酸酐和酯的缩合反应; 通过酰腙与各种亲核试剂的加成
反应也是制备酰肼类化合物的重要方法 ${ }^{[7]}$.

含三氟甲基的有机化合物由于具有独特的物理化 学性质而被广泛应用于医药、农药和材料科学等领域 ${ }^{[8]}$. 目前，向有机物分子中引入三氟甲基的方法，除了直接 使用三氟甲基化试剂外，利用含有三氟甲基的有机小分 子作为合成砌块也是一种行之有效的简便方法 ${ }^{[9,10]}$. 在 探索三氟甲基化反应过程中, 我们小组发展了以工业上 价廉易得、稳定的三氟甲基酰腙作为三氟甲基合成砌块, 探索了该酰腙参与的 1,2-加成反应 ${ }^{[11]}$ 和 $[3+2]$ 环加成反 应 $^{[12]}$, 取得了较好反应结果. 同时，由于酰腙的氰基化 反应是制备 $\alpha$-肼基羧酸的重要方法之一，而后者可作为 一些氨基酸代谢酶的抑制剂以及用于非天然肽的设计 合成中 ${ }^{[13]}$. 因此，本文报道以三甲基氭硅烷作为氰基化 试剂，探索了三氟甲基酰腙的氧基化反应，合成了一系

* Corresponding author. E-mail: huyl@nwnu.edu.cn

Received October 11, 2019; revised December 10, 2019; published online December 27, 2019.

Project supported by the National Natural Science Foundation of China (Nos. 21662030, 21861033).

国家自然科学基金(Nos. 21662030, 21861033)资助项目. 
<smiles>CC(C)(C)N(NC(=O)c1ccccc1)C(=O)c1ccccc1</smiles>

$\mathrm{RH}-5849$

A<smiles>CCc1ccc(C(=O)NN(C(=O)c2cc(C)cc(C)c2)C(C)(C)C)cc1</smiles>

Tebufenozide (RH-5992)

B<smiles>COc1cccc(C(=O)NN(C(=O)c2cc(C)cc(C)c2)C(C)(C)C)c1C</smiles>

Methoxyfenozide (RH-2485)

C<smiles>CC(C)(C)N(NC(=O)c1ccc(Cl)cc1)C(=O)c1ccccc1</smiles>

Halofenozide (RH-0345)

D<smiles>Cc1cc(C)cc(C(=O)N(NC(=O)c2ccc3c(c2C)CCCO3)C(C)(C)C)c1</smiles>

Chromafenozide (ANS-118)

E

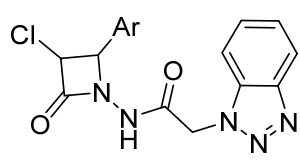

Antibacterial agent

$F$<smiles>CC(C)(C)c1cc(/C=N/NC(=O)c2cc3c(cc2[N+](=O)[O-])OCO3)cc(C(C)(C)C)c1O</smiles>

Anti-inflammatory agent (LASSBio-881)

G

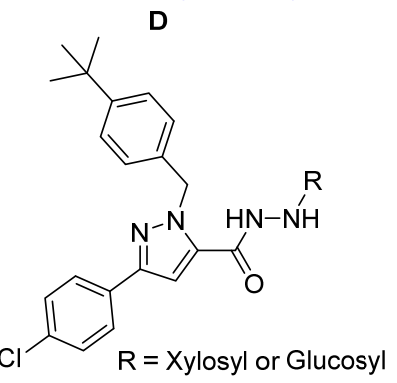

Anticancer agents<smiles>[X][X]C([R])C(=O)NNC(C)C(CC)(CC)C(=O)NC([R])CC(=O)OC</smiles>

I

图 1 具有生物活性的酰肼化合物

Figure 1 Bioactive compound of hydrazides

列含三氟甲基的氧基酰肼类化合物，进一步拓展三氟甲 基酰腙在三氟甲基化反应中的应用范围.

\section{1 结果与讨论}

\section{1 路易斯酸催化下三氟甲基酰腙与三甲基氰硅烷的 氭基化反应研究}

首先, 在二氯甲烷溶剂中, 尝试了三氟甲基酰腙 (1a) (1 equiv.)与三甲基氰硅烷(2) (2.5 equiv.)的氰基化反 应. 结果发现, 在室温条件下反应没有发生. 为了促进 三甲基氰硅烷释放氧根负离子, 在反应中加入了氟化钾 (2.5 equiv.), 但是反应依然没有发生. 于是将该反应在 三氟化硼乙醚 $(0.2$ equiv.)作用下进行, 反应 $60 \mathrm{~h}$ 后以 $15 \%$ 的收率得到了目标产物 $\mathbf{3 a}$ (表 1, Entries 1 3). 为了 进一步提高 $3 \mathrm{a}$ 的产率, 其他路易斯酸如三氯化铝、四氯 化钛和三氟甲磺酸盐等也被用于该反应中(表 1 , Entries 4 11). 研究表明, 在三氟甲磺酸铟作用下, 化合物 3a 的产率最高, 可达 $85 \%$ (表 1, Entry 7). 通过对反应温度 的调节, 发现升高温度对反应有利: 当该反应在回流条 件下进行时，化合物 3a 的产率可提高到 $91 \%$, 并且反应 时间缩短到 $45 \mathrm{~h}$ (表 1, Entries 12 和 13). 在此条件下考察 了各种溶剂对该反应的影响. 结果发现, 三氯甲烷、1,2二氯乙烷和乙腈等极性溶剂对反应也是有利的(表 1 , Entries 14 16); 非极性溶剂如四氢呋喃、1,4-二氧六环 对该反应不利(表 1, Entries 17 和 18); 当反应在质子溶
剂如甲醇中进行时, 化合物 3a 的产率大大降低(表 1, Entry 19). 最后, 对该反应的物料比进行了考察(表 1, Entries 20 23). 研究表明，三氟甲基酰腙(1a)、三甲基 氧硅烷(2)和三氟甲磺酸铟的物质的量的比为 $1: 2.5$ : 0.2 时, $\mathbf{3 a}$ 的产率最高(表 1, Entry 13). 为了进一步缩短 反应时间，在上述反应条件下分别尝试了在反应体系中 加入氟化钾、三乙胺和甲醇 ${ }^{[13 b]}$ 的反应, 结果均不理想 (表 1, Entries 24〜26).

综上所述，合成化合物 $\mathbf{3 a}$ 的最佳反应条件为: 三氟 甲基酰腙(1a)、三甲基氰硅烷(2)、三氟甲磺酸铟的物质 的量的比为 $1: 2.5: 0.2$, 溶剂为二氯甲烷, 回流条件, 反应时间为 $45 \mathrm{~h}$.

为了探索该反应的适应性，在上述最佳反应条件 下，对各种三氟甲基酰腙与三甲基氰硅烷的反应情况进 行了考察(表 2). 由表 2 可知, 对于苯环上连有取代基的 三氟甲基酰腙，不管是吸电子的还是供电子的，反应都 能顺利进行，所得产物的产率在 $62 \% \sim 93 \%$ 之间(3b $3 \mathbf{k}$ ). 研究还发现，取代基在酰腙分子中苯环上的位置 对反应有一定影响. 例如, 当甲基在苯环对位上的酰腙 作为底物时，所得产物 $\mathbf{3 d}$ 的产率为 $78 \%$ ，而当甲基在苯 环邻位和间位上的酰腙作为底物时, 所得产物 $\mathbf{3 b}$ 和 $3 \mathrm{c}$ 的产率分别为 $93 \%$ 和 $86 \%$. 相比之下, 苯环对位上连有 氯原子和溴原子的酰腙作为底物时, 所得产物的产率较 低, 分别为 $76 \%$ 和 $62 \%(3 \mathrm{~h} \sim 3 \mathbf{i})$. 当使用菜酰肼形成的 
表 1 合成化合物 $\mathbf{3 a}$ 的反应条件优化 ${ }^{a}$

Table 1 Optimization of reaction condition for the synthesis of compound 3a<smiles>N#CC(NNC(=O)c1ccccc1)C(F)(F)F</smiles>

\begin{tabular}{|c|c|c|c|c|c|c|}
\hline Entry & Mole ratio of $1 \mathbf{a} / \mathbf{2} /$ Cat. & Catalyst & Solvent & Temperature & Time/h & Isolated yield/ $\%$ \\
\hline 1 & $1 / 2.5 / 0$ & - & DCM & r.t & 60 & 0 \\
\hline $2^{b}$ & $1 / 2.5 / 0$ & - & DCM & r.t & 60 & 0 \\
\hline 3 & $1 / 2.5 / 0.2$ & $\mathrm{BF}_{3} \cdot \mathrm{OEt}_{2}$ & DCM & r.t & 60 & 15 \\
\hline 4 & $1 / 2.5 / 0.2$ & $\mathrm{AlCl}_{3}$ & DCM & r.t & 60 & 36 \\
\hline 5 & $1 / 2.5 / 0.2$ & $\mathrm{TiCl}_{4}$ & DCM & r.t & 60 & 63 \\
\hline 6 & $1 / 2.5 / 0.2$ & $\mathrm{Sc}(\mathrm{OTf})_{3}$ & DCM & r.t & 60 & 0 \\
\hline 7 & $1 / 2.5 / 0.2$ & $\operatorname{In}(\mathrm{OTf})_{3}$ & DCM & r.t & 60 & 85 \\
\hline 8 & $1 / 2.5 / 0.2$ & $\mathrm{Yb}(\mathrm{OTf})_{3}$ & DCM & r.t & 60 & 48 \\
\hline 9 & $1 / 2.5 / 0.2$ & $\mathrm{Fe}(\mathrm{OTf})_{3}$ & DCM & r.t & 60 & 0 \\
\hline 10 & $1 / 2.5 / 0.2$ & $\mathrm{Hf}(\mathrm{OTf})_{3}$ & DCM & r.t & 60 & 0 \\
\hline 11 & $1 / 2.5 / 0.2$ & $\mathrm{Ni}(\mathrm{OTf})_{3}$ & DCM & r.t & 60 & 0 \\
\hline 12 & $1 / 2.5 / 0.2$ & $\operatorname{In}(\mathrm{OTf})_{3}$ & DCM & -5 & 60 & 4 \\
\hline 13 & $1 / 2.5 / 0.2$ & $\operatorname{In}(\mathrm{OTf})_{3}$ & DCM & Reflux & 45 & 91 \\
\hline 14 & $1 / 2.5 / 0.2$ & $\operatorname{In}(\mathrm{OTf})_{3}$ & DCE & Reflux & 10 & 71 \\
\hline 15 & $1 / 2.5 / 0.2$ & $\operatorname{In}(\mathrm{OTf})_{3}$ & $\mathrm{CHCl}_{3}$ & Reflux & 45 & 73 \\
\hline 16 & $1 / 2.5 / 0.2$ & $\operatorname{In}(\mathrm{OTf})_{3}$ & $\mathrm{CH}_{3} \mathrm{CN}$ & Reflux & 45 & 67 \\
\hline 17 & $1 / 2.5 / 0.2$ & $\operatorname{In}(\mathrm{OTf})_{3}$ & THF & Reflux & 45 & 45 \\
\hline 18 & $1 / 2.5 / 0.2$ & $\operatorname{In}(\mathrm{OTf})_{3}$ & 1,4-Dioxane & Reflux & 45 & 33 \\
\hline 19 & $1 / 2.5 / 0.2$ & $\operatorname{In}(\mathrm{OTf})_{3}$ & $\mathrm{MeOH}$ & Reflux & 45 & 8 \\
\hline 20 & $1 / 2.5 / 0.3$ & $\operatorname{In}(\mathrm{OTf})_{3}$ & DCM & Reflux & 30 & 66 \\
\hline 21 & $1 / 2.5 / 0.1$ & $\operatorname{In}(\mathrm{OTf})_{3}$ & DCM & Reflux & 45 & 16 \\
\hline 22 & $1 / 1.5 / 0.2$ & $\operatorname{In}(\mathrm{OTf})_{3}$ & DCM & Reflux & 45 & 55 \\
\hline 23 & $1 / 3.5 / 0.2$ & $\operatorname{In}(\mathrm{OTf})_{3}$ & DCM & Reflux & 40 & 84 \\
\hline $24^{b}$ & $1 / 2.5 / 0.2$ & $\operatorname{In}(\mathrm{OTf})_{3}$ & DCM & Reflux & 45 & 0 \\
\hline $25^{c}$ & $1 / 2.5 / 0.2$ & $\operatorname{In}(\mathrm{OTf})_{3}$ & DCM & Reflux & 45 & 0 \\
\hline $26^{d}$ & $1 / 2.5 / 0.2$ & $\operatorname{In}(\mathrm{OTf})_{3}$ & DCM & Reflux & 45 & Trace \\
\hline
\end{tabular}

${ }^{a}$ All reactions were carried out by using $0.2 \mathrm{mmol}$ of $\mathbf{1 a}$, solvent $(3.0 \mathrm{~mL}) .{ }^{b} \mathrm{KF}(2.5$ equiv. $)$ was added. ${ }^{c}\left(\mathrm{C}_{2} \mathrm{H}_{5}\right)_{3} \mathrm{~N}(2.5$ equiv. $)$ was added. ${ }^{d} \mathrm{CH}_{3} \mathrm{OH}(2.5$ equiv. $)$ was added.

三氟甲基酰腙作为底物时，虽然反应时间有所延长，但 反应也能发生, 31 的产率可达 $82 \%$. 当使用 2-呋喃酰肼 形成的三氟甲基酰腙作为底物时, 反应也能顺利进行, 但是产率降低, $3 \mathrm{~m}$ 的产率只有 $60 \%$. 当 $\mathrm{R}^{1}$ 为脂肪族烃 基, 如异丙基、叔丁基、环已基和苄基时, 反应依然能 够顺利发生, 但是所得产物的产率比 $\mathrm{R}^{1}$ 为芳基时的要 低( $3 n \sim 3 q)$. 研究表明, 当 $R^{1}$ 为直链烷基时, 碳链的长 短对反应有影响. 当 $\mathrm{R}^{1}$ 为长链烷基如月桂基时, 所得产 物 $3 \mathrm{r}$ 的产率高达 $90 \%$; 当 $\mathrm{R}^{1}$ 为短链烷基如正丙基时, 没有得到目标产物 $3 \mathrm{~s}$. 对于这种反应活性差异还需进一 步研究. 最后，尝试了三氟甲基酮酰腙的㲵基化反应， 反应没有发生 $(\mathbf{3 t} \sim 3 \mathbf{u})$, 这是由于酮酰腙的活性比醛酰 腙的活性差的缘故.

为了探索该反应的实用性, 在上述最佳反应条件 下, 对三氟甲基酰腙 $1 \mathrm{a}$ 的氰基化反应进行了克级规模 反应. 结果表明反应也能顺利进行, 并以 $85 \%$ 的产率得 到了 3a (Eq. 1).

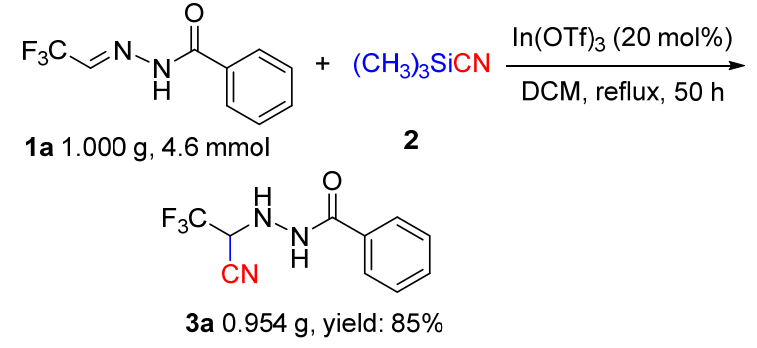

在上述研究基础上，对三氟甲基酰腙的氰基化反应 产物也进行了官能团转化研究. 例如将 $\mathbf{3 a}$ 或 $\mathbf{3 b}$ 在盐酸 甲醇中回流, 都能顺利地发生水解/酯化反应, 得到 $\alpha$-肼 基氨基酸酯 7a 或 $7 \mathbf{b}$ (Eq. 2), 为进一步合成具有潜在应 用的含三氟甲基的 $\alpha$-肼基羧酸衍生物提供了依据.

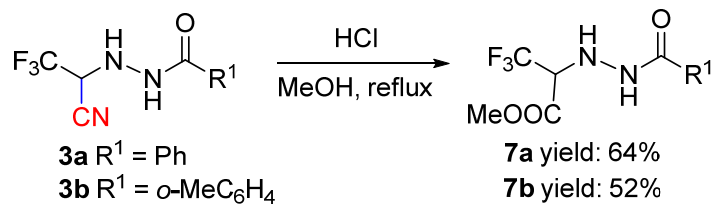


表 2 三氟甲基酰腙的氰基化反应 ${ }^{a}$

Table 2 Hydrocyanation reactions of trifluoromethylated $N$-acylhydrazones

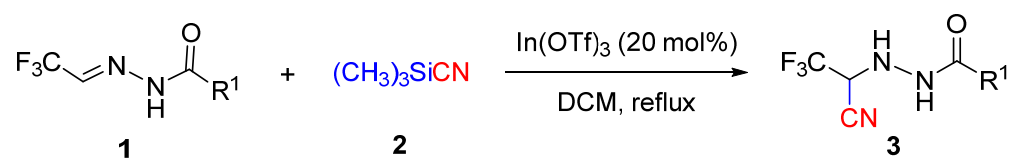<smiles>N#CC(NNC(=O)c1ccccc1)C(F)(F)F</smiles><smiles>Cc1ccccc1C(=O)NNC(C#N)C(F)(F)F</smiles>

$3 a$ $45 \mathrm{~h}$, yield: $91 \%^{b}$<smiles>N#CC(NNC(=O)c1ccc(Cl)cc1)C(F)(F)F</smiles>

$3 \mathrm{~h}$ $28 \mathrm{~h}$, yield: $76 \%$<smiles>CC(C)(C)C(=O)NNC(C#N)C(F)(F)F</smiles>

$$
3 \mathbf{i}
$$

$\mathbf{3 b}$

$15 \mathrm{~h}$, yield: $93 \%$<smiles>N#CC(NNC(=O)c1ccc(Br)cc1)C(F)(F)F</smiles>

$30 \mathrm{~h}$, yield: $62 \%$<smiles>N#CC(NNC(=O)C1CCCCC1)C(F)(F)F</smiles><smiles>Cc1cccc(C(=O)NNC(C#N)C(F)(F)F)c1</smiles>

$3 c$ $14 \mathrm{~h}$, yield: $86 \%$<smiles>N#CC(NNC(=O)c1ccc(F)cc1)C(F)(F)F</smiles>

$3 \mathbf{j}$ $45 \mathrm{~h}$, yield: $92 \%$<smiles>N#CC(NNC(=O)Cc1ccccc1)C(F)(F)F</smiles>

$3 q$<smiles>Cc1ccc(C(=O)NNC(C#N)C(F)(F)F)cc1</smiles>

3d

$15 \mathrm{~h}$, yield: $78 \%$<smiles>N#CC(NNC(=O)c1ccc(C(F)(F)F)cc1)C(F)(F)F</smiles>

$3 \mathbf{k}$

$50 \mathrm{~h}$, yield: $84 \%$

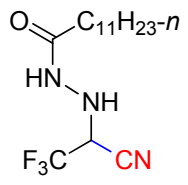

$3 r$<smiles>COc1ccc(C(=O)NNC(C#N)C(F)(F)F)cc1</smiles>

$3 e$ $45 \mathrm{~h}$, yield: $92 \%$<smiles>N#CC(NNC(=O)c1ccc2ccccc2c1)C(F)(F)F</smiles>

$3 \mathbf{1}$

$50 \mathrm{~h}$, yield: $82 \%$<smiles>CCCC(=O)NNC(C#N)C(F)(F)F</smiles>

$3 \mathrm{~s}$<smiles>N#CC(NNC(=O)c1ccccc1Cl)C(F)(F)F</smiles>

$3 f$

$30 \mathrm{~h}$, yield: $83 \%$<smiles>N#CC(NNC(=O)c1ccco1)C(F)(F)F</smiles>

$3 m$

$24 \mathrm{~h}$, yield: $60 \%$<smiles>CC(C#N)(NNC(=O)c1ccccc1)C(F)(F)F</smiles>

$3 \mathrm{t}$

$45 \mathrm{~h}$, yield: $0 \%$<smiles>N#CC(NNC(=O)c1cccc(Cl)c1)C(F)(F)F</smiles>

$3 \mathrm{~g}$

$24 \mathrm{~h}$, yield: $83 \%$<smiles>CC(C)C(=O)NNC(C#N)C(F)(F)F</smiles>

$3 n$ $24 \mathrm{~h}$, yield: $67 \%$

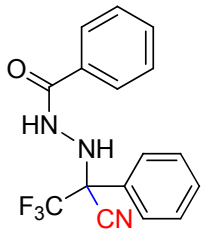

3u

$45 \mathrm{~h}$, yield: $0 \%$

${ }^{a}$ All reactions were carried out by using $0.1 \mathrm{mmol}$ of $\mathbf{1}, \mathrm{DCM}(3.0 \mathrm{~mL})$, stirred at reflux temperature, isolated yield. ${ }^{b}$ This reaction was carried out by using $0.2 \mathrm{mmo}$ of $1 \mathbf{a}$.

\section{2 反应机理探讨}

在文献报道 ${ }^{[7 \mathrm{c}]}$ 和实验结果基础上, 我们认为在三氟 甲磺酸铟催化下，三氟甲基酰腙的氰基化反应机理可能 如 Scheme 1 所示: 首先，三氟甲基酰腙 $\mathbf{1}$ 和三氟甲磺酸 铟(4)形成络合物 5; 然后，三甲基氰硅烷(2)进攻络合物 5 生成 $\mathrm{Si}-\mathrm{N}$ 中间体 6 , 同时脱去三氟甲磺酸铟(4)继续进 行反应; 最后, $\mathrm{Si}-\mathrm{N}$ 中间体 $\mathbf{6}$ 经水解生成化合物 $\mathbf{3}$, 并伴 随有三甲基硅醇生成.

\section{2 结论}

综上所述，本文报道了在三氟甲磺酸铟催化下，以 二氯甲烷为溶剂, 在回流条件下, 通过三氟甲基酰腙和 三甲基氰硅烷的 1,2-加成反应来合成含有三氟甲基的氰 基酰肼类化合物的方法. 该方法以价廉、稳定、易得的 三氟甲基酰腙作为三氟甲基合成砌块, 实现了它的氰基 化反应，进一步拓展了三氟甲基酰腙在合成含三氟甲基 化合物中的应用范围.

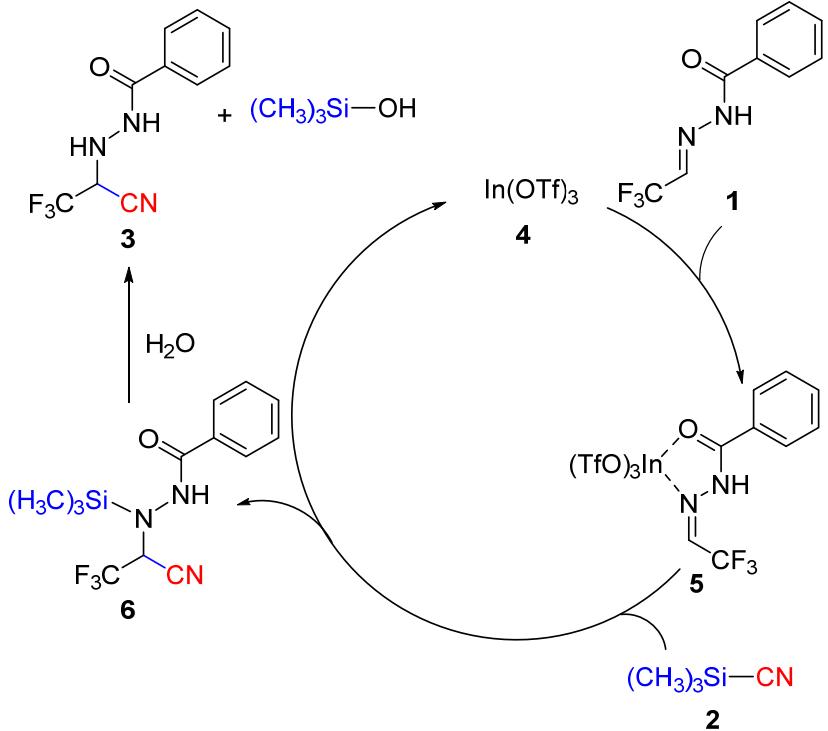

图式 1 可能的反应机理

Scheme 1 Proposed mechanism 


\section{3 实验部分}

\section{1 仪器与试剂}

红外光谱仪为 Digilab FTS 3000, 固体采用 $\mathrm{KBr}$ 压 片法, 液体采用液膜法; 核磁共振谱用 BRUKER PT jxf790425AM $400 \mathrm{MHz} / 600 \mathrm{MHz}$ 型核磁共振仪测定, 以 氛代氯仿作为溶剂, TMS 为内标; 高分辨质谱用 Bruker APEX II 傅里叶变换离子回旋共振质谱仪测定, ESI 源; 熔点用显微熔点测定仪测定, 温度未校正. 实验中所用 试剂均为国产分析纯级. 所用溶剂均通过标准方法蒸馏 干燥.

\section{2 实验方法}

\subsection{1 化合物 $\mathbf{3}$ 的合成}

在 $50 \mathrm{~mL}$ 的圆底烧瓶中依次加入三氟甲基酰腙(1a) $(0.043 \mathrm{~g}, 0.2 \mathrm{mmol})$ 、三甲基氧硅烷(2) $(0.050 \mathrm{~g}, 0.5$ $\mathrm{mmol}) 、$ 三氟甲磺酸铟 $(0.022 \mathrm{~g}, 0.04 \mathrm{mmol})$ 和 $3 \mathrm{~mL}$ 二氯 甲烷(DCM), 将此混合液在回流条件下摚拌, 薄层色谱 (TLC) 检测反应, $45 \mathrm{~h}$ 反应完全. 向反应混合物中加入 3 $\mathrm{mL}$ 饱和碳酸氢钠溶液, 搅拌 $10 \mathrm{~min}$, 用乙酸乙酯萃取 $(5$ $\mathrm{mL} \times 3)$, 分出有机相, 用无水硫酸镁干燥. 蒸去溶剂后 进行柱层析 $[V($ 石油醚 $): V($ 乙酸乙酯 $)=5: 1]$ 分离得产 物 $N^{\prime}$-(1,1,1-三氟丙腈-2-基)苯甲酰肼(3a) $44 \mathrm{mg}$, 产率 $91 \%$. 白色固体, m.p. $113 \sim 115{ }^{\circ} \mathrm{C} ;{ }^{1} \mathrm{H}$ NMR $(600 \mathrm{MHz}$, $\left.\mathrm{CDCl}_{3}\right) \delta: 7.96(\mathrm{~s}, 1 \mathrm{H}), 7.79(\mathrm{~d}, J=7.8 \mathrm{~Hz}, 2 \mathrm{H}), 7.58(\mathrm{t}$, $J=7.2 \mathrm{~Hz}, 1 \mathrm{H}), 7.47(\mathrm{t}, J=7.8 \mathrm{~Hz}, 2 \mathrm{H}), 5.77(\mathrm{~s}, 1 \mathrm{H})$, $4.69 \sim 4.65(\mathrm{~m}, 1 \mathrm{H}) ;{ }^{13} \mathrm{C}$ NMR $\left(150 \mathrm{MHz}, \mathrm{CDCl}_{3}\right) \delta$ : 168.8, 133.1, 131.1, 129.1, 127.4, 121.6 (q, $J=280.1 \mathrm{~Hz})$, $111.6,54.9$ (q, $J=33.2 \mathrm{~Hz}) ;{ }^{19} \mathrm{~F}$ NMR $\left(376 \mathrm{MHz}, \mathrm{CDCl}_{3}\right)$ $\delta:-73.32$ (d, $J=6.4 \mathrm{~Hz})$; IR (KBr) v: 3464, 3397, 2951, $1646,1538,1471,1262,1146 \mathrm{~cm}^{-1}$; HRMS (ESI) calcd for $\mathrm{C}_{10} \mathrm{H}_{8} \mathrm{~F}_{3} \mathrm{~N}_{3} \mathrm{ONa}[\mathrm{M}+\mathrm{Na}]^{+}$266.0512, found 266.0518.

化合物 3b $\sim 3 r$ 的合成方法同 3a.

$N^{\prime}$-(1,1,1-三氟丙腈-2-基)-2-甲基苯甲酰肼(3b): 24 $\mathrm{mg}$, 产率 93\%. 白色固体, m.p. 108 110 ${ }^{\circ} \mathrm{C} ;{ }^{1} \mathrm{H}$ NMR $\left(600 \mathrm{MHz}, \mathrm{CDCl}_{3}\right) \delta: 7.60(\mathrm{~s}, 1 \mathrm{H}), 7.41(\mathrm{t}, J=7.8 \mathrm{~Hz}, 1 \mathrm{H})$, $7.38(\mathrm{~d}, J=7.8 \mathrm{~Hz}, 1 \mathrm{H}), 7.26(\mathrm{~d}, J=6.6 \mathrm{~Hz}, 1 \mathrm{H}), 7.23(\mathrm{t}$, $J=7.2 \mathrm{~Hz}, 1 \mathrm{H}), 5.88(\mathrm{~s}, 1 \mathrm{H}), 4.71 \sim 4.67(\mathrm{~m}, 1 \mathrm{H}), 2.47$ (s, $3 \mathrm{H}) ;{ }^{13} \mathrm{C}$ NMR $\left(150 \mathrm{MHz}, \mathrm{CDCl}_{3}\right) \delta: 170.7,137.5,132.1$, 131.6, 131.5, 127.4, 126.1, 121.7 (q, $J=279.6 \mathrm{~Hz}), 111.6$, 54.8 (q, $J=33.3 \mathrm{~Hz}), 19.9 ;{ }^{19} \mathrm{~F} \mathrm{NMR}\left(376 \mathrm{MHz}, \mathrm{CDCl}_{3}\right) \delta$ : $-73.32(\mathrm{~d}, J=6.8 \mathrm{~Hz}$ ); IR (KBr) $v: 3439,2951,1645$, 1537, 1514, 1459, 1262, 1199, $1150 \mathrm{~cm}^{-1}$; HRMS (ESI) calcd for $\mathrm{C}_{11} \mathrm{H}_{10} \mathrm{~F}_{3} \mathrm{~N}_{3} \mathrm{ONa}[\mathrm{M}+\mathrm{Na}]^{+} 280.0668$, found 280.0661 .

$N^{\prime}$-(1,1,1-三氟丙腈-2-基)-3-甲基苯甲酰肼(3c)：22 $\mathrm{mg}$, 产率 $86 \%$. 白色固体, m.p. $114 \sim 115{ }^{\circ} \mathrm{C} ;{ }^{1} \mathrm{H}$ NMR $\left(600 \mathrm{MHz}, \mathrm{CDCl}_{3}\right) \delta: 7.93(\mathrm{~s}, 1 \mathrm{H}), 7.60(\mathrm{~s}, 1 \mathrm{H}), 7.56(\mathrm{~d}$, $J=7.2 \mathrm{~Hz}, 1 \mathrm{H}), 7.38(\mathrm{~d}, J=7.2 \mathrm{~Hz}, 1 \mathrm{H}), 7.34$ (t, $J=7.8$ $\mathrm{Hz}, 1 \mathrm{H}), 5.76(\mathrm{t}, J=4.8 \mathrm{~Hz}, 1 \mathrm{H}), 4.68 \sim 4.64(\mathrm{~m}, 1 \mathrm{H}), 2.39$ $(\mathrm{s}, 3 \mathrm{H}) ;{ }^{13} \mathrm{C}$ NMR $\left(150 \mathrm{MHz}, \mathrm{CDCl}_{3}\right) \delta: 169.0,139.1$, 133.8, 131.1, 129.0, 128.0, 124.4, 121.6 (q, $J=280.2 \mathrm{~Hz}$ ), 111.6, 55.0 (q, $J=33.0 \mathrm{~Hz}), 21.4 ;{ }^{19} \mathrm{~F}$ NMR $(376 \mathrm{MHz}$, $\left.\mathrm{CDCl}_{3}\right) \delta:-73.29(\mathrm{~d}, J=6.4 \mathrm{~Hz})$; IR (KBr) $v: 3440$, 2925, 1652, 1536, 1514, 1464, 1260, 1198, $1149 \mathrm{~cm}^{-1}$; HRMS (ESI) calcd for $\mathrm{C}_{11} \mathrm{H}_{10} \mathrm{~F}_{3} \mathrm{~N}_{3} \mathrm{ONa}[\mathrm{M}+\mathrm{Na}]^{+}$ 280.0668 , found 280.0662 .

$N^{\prime}$-(1,1,1-三氟丙腈-2-基)-4-甲基苯甲酰肼(3d)：20 $\mathrm{mg}$, 产率 78\%. 白色固体, m.p. $124 \sim 126{ }^{\circ} \mathrm{C} ;{ }^{1} \mathrm{H}$ NMR $\left(600 \mathrm{MHz}, \mathrm{CDCl}_{3}\right) \delta: 7.80(\mathrm{~s}, 1 \mathrm{H}), 7.68(\mathrm{~d}, J=7.8 \mathrm{~Hz}$, $2 \mathrm{H}), 7.27(\mathrm{~d}, J=9.0 \mathrm{~Hz}, 2 \mathrm{H}), 5.67(\mathrm{t}, J=5.4 \mathrm{~Hz}, 1 \mathrm{H})$, $4.66 \sim 4.62(\mathrm{~m}, 1 \mathrm{H}), 2.42(\mathrm{~s}, 3 \mathrm{H}) ;{ }^{13} \mathrm{C}$ NMR $(150 \mathrm{MHz}$, $\left.\mathrm{CDCl}_{3}\right) \delta: 168.7,143.9,129.8,128.2,127.4,121.6$ (q, $J=$ $280.4 \mathrm{~Hz}), 111.6,55.0$ (q, $J=33.0 \mathrm{~Hz}), 21.7 ;{ }^{19} \mathrm{~F}$ NMR $\left(376 \mathrm{MHz}, \mathrm{CDCl}_{3}\right) \delta:-73.31(\mathrm{~d}, J=6.4 \mathrm{~Hz}) ; \mathrm{IR}(\mathrm{KBr}) v$ : 3441, 3262, 2960, 1647, 1537, 1473, 1267, 1197, 1150, $1116 \mathrm{~cm}^{-1}$; HRMS (ESI) calcd for $\mathrm{C}_{11} \mathrm{H}_{10} \mathrm{~F}_{3} \mathrm{~N}_{3} \mathrm{ONa}[\mathrm{M}+$ $\mathrm{Na}]^{+} 280.0668$, found 280.0658 .

$N^{\prime}$-(1,1,1-三氟丙腈-2-基)-4-甲氧基苯甲酰肼(3e): 25 $\mathrm{mg}$, 产率 92\%. 白色固体, m.p. $136 \sim 137{ }^{\circ} \mathrm{C} ;{ }^{1} \mathrm{H}$ NMR $\left(600 \mathrm{MHz}, \mathrm{CDCl}_{3}\right) \delta: 7.76(\mathrm{~d}, J=9.0 \mathrm{~Hz}, 2 \mathrm{H}), 7.73(\mathrm{~s}$, $1 \mathrm{H}), 6.95(\mathrm{~d}, J=9.0 \mathrm{~Hz}, 2 \mathrm{H}), 5.65(\mathrm{t}, J=5.4 \mathrm{~Hz}, 1 \mathrm{H})$, $4.65 \sim 4.61(\mathrm{~m}, 1 \mathrm{H}), 3.87(\mathrm{~s}, 3 \mathrm{H}) ;{ }^{13} \mathrm{C}$ NMR $(150 \mathrm{MHz}$, $\left.\mathrm{CDCl}_{3}\right) \delta: 168.3,163.5,129.4,123.1,121.6(\mathrm{q}, J=280.4$ $\mathrm{Hz}), 114.4,110.2,55.7,55.0$ (q, $J=33.0 \mathrm{~Hz}) ;{ }^{19} \mathrm{~F}$ NMR $\left(376 \mathrm{MHz}, \mathrm{CDCl}_{3}\right) \delta:-73.32(\mathrm{~d}, J=6.0 \mathrm{~Hz})$; IR (KBr) $v$ : $3439,2929,1644,1612,1543,1516,1461,1261,1192$, $1150 \mathrm{~cm}^{-1}$; HRMS (ESI) calcd for $\mathrm{C}_{11} \mathrm{H}_{10} \mathrm{~F}_{3} \mathrm{~N}_{3} \mathrm{O}_{2} \mathrm{Na}[\mathrm{M}+$ $\mathrm{Na}]^{+}$296.0617, found 296.0608.

$N^{\prime}$-(1,1,1-三氟丙腈-2-基)-2-氯苯甲酰肼(3f): $23 \mathrm{mg}$, 产率 $83 \%$. 白色固体, m.p. $107 \sim 109{ }^{\circ} \mathrm{C} ;{ }^{1} \mathrm{H}$ NMR $(600$ $\left.\mathrm{MHz}, \mathrm{CDCl}_{3}\right) \delta: 8.01(\mathrm{~s}, 1 \mathrm{H}), 7.72(\mathrm{~d}, J=7.8 \mathrm{~Hz}, 1 \mathrm{H})$, $7.45(\mathrm{~d}, J=3.6 \mathrm{~Hz}, 2 \mathrm{H}), 7.39 \sim 7.36(\mathrm{~m}, 1 \mathrm{H}), 5.77(\mathrm{t}, J=$ $4.2 \mathrm{~Hz}, 1 \mathrm{H}), 4.72 \sim 4.68(\mathrm{~m}, 1 \mathrm{H}) ;{ }^{13} \mathrm{C}$ NMR $(150 \mathrm{MHz}$, $\left.\mathrm{CDCl}_{3}\right) \delta: 167.5,132.8,131.4,131.3,130.8,130.7,127.5$, 121.6 (q, $J=280.2 \mathrm{~Hz}), 111.5,54.7(\mathrm{q}, J=33.3 \mathrm{~Hz}) ;{ }^{19} \mathrm{~F}$ NMR $\left(376 \mathrm{MHz}, \mathrm{CDCl}_{3}\right) \delta:-73.35(\mathrm{~d}, J=6.4 \mathrm{~Hz})$; IR (KBr) $v: 3440,2970,2926,1649,1541,1460,1263,1197$, $1149 \mathrm{~cm}^{-1}$; HRMS (ESI) calcd for $\mathrm{C}_{10} \mathrm{H}_{7} \mathrm{ClF}_{3} \mathrm{~N}_{3} \mathrm{ONa}[\mathrm{M}+$ $\mathrm{Na}]^{+}$300.0122, found 300.0117.

$N^{\prime}$-(1,1,1-三氟丙腈-2-基)-3-氯苯甲酰肼(3g): $23 \mathrm{mg}$, 
产率 $83 \%$. 白色固体, m.p. $131 \sim 132{ }^{\circ} \mathrm{C} ;{ }^{1} \mathrm{H}$ NMR (600 $\left.\mathrm{MHz}, \mathrm{CDCl}_{3}\right) \delta: 7.84(\mathrm{~s}, 1 \mathrm{H}), 7.78(\mathrm{~s}, 1 \mathrm{H}), 7.66(\mathrm{~d}, J=7.2$ $\mathrm{Hz}, 1 \mathrm{H}), 7.56(\mathrm{~d}, J=7.8 \mathrm{~Hz}, 1 \mathrm{H}), 7.42(\mathrm{t}, J=7.8 \mathrm{~Hz}, 1 \mathrm{H})$, $5.67(\mathrm{~s}, 1 \mathrm{H}), 4.66 \sim 4.62(\mathrm{~m}, 1 \mathrm{H}) ;{ }^{13} \mathrm{C}$ NMR $(150 \mathrm{MHz}$, $\left.\mathrm{CDCl}_{3}\right) \delta: 167.5,135.5,133.2,130.5,127.8,125.4,121.5$ (q, $J=280.4 \mathrm{~Hz}), 111.4,54.9$ (q, $J=33.2 \mathrm{~Hz}) ;{ }^{19} \mathrm{~F}$ NMR $\left(376 \mathrm{MHz}, \mathrm{CDCl}_{3}\right) \delta:-73.27(\mathrm{~d}, J=6.4 \mathrm{~Hz})$; IR $(\mathrm{KBr}) v$ : $3427,2925,1654,1534,1461,1263,1199,1150 \mathrm{~cm}^{-1}$; HRMS (ESI) calcd for $\mathrm{C}_{10} \mathrm{H}_{7} \mathrm{ClF}_{3} \mathrm{~N}_{3} \mathrm{ONa}[\mathrm{M}+\mathrm{Na}]^{+}$ 300.0122, found 300.0107.

$N^{\prime}$-(1,1,1-三氟丙腈-2-基)-4-氯苯甲酰肼(3h): $21 \mathrm{mg}$, 产率 76\%. 白色固体, m.p. $116 \sim 117{ }^{\circ} \mathrm{C} ;{ }^{1} \mathrm{H}$ NMR $(600$ $\left.\mathrm{MHz}, \mathrm{CDCl}_{3}\right) \delta: 7.80(\mathrm{~s}, 1 \mathrm{H}), 7.73(\mathrm{~d}, J=9.0 \mathrm{~Hz}, 2 \mathrm{H})$, $7.46(\mathrm{~d}, J=8.4 \mathrm{~Hz}, 2 \mathrm{H}), 5.66(\mathrm{t}, J=4.8 \mathrm{~Hz}, 1 \mathrm{H}), 4.66 \sim$ $4.62(\mathrm{~m}, 1 \mathrm{H}) ;{ }^{13} \mathrm{C}$ NMR $\left(150 \mathrm{MHz}, \mathrm{CDCl}_{3}\right) \delta: 167.8$, 139.7, 129.5, 129.4, 128.8, 121.6 (q, $J=280.2 \mathrm{~Hz}$ ), 111.5, 54.9 (q, $J=33.3 \mathrm{~Hz}) ;{ }^{19} \mathrm{~F}$ NMR $\left(376 \mathrm{MHz}, \mathrm{CDCl}_{3}\right) \delta$ : -73.28 (d, $J=6.0 \mathrm{~Hz}$ ); IR (KBr) v: 3426, 3315, 2927, 1647, 1535, 1514, 1475, 1261, 1203, $1153 \mathrm{~cm}^{-1}$; HRMS (ESI) calcd for $\mathrm{C}_{10} \mathrm{H}_{7} \mathrm{ClF}_{3} \mathrm{~N}_{3} \mathrm{ONa}[\mathrm{M}+\mathrm{Na}]^{+} 300.0122$, found 300.0109 .

$N^{\prime}$-(1,1,1-三氟丙腈-2-基)-4-溴苯甲酰肼(3i): $20 \mathrm{mg}$, 产率 $62 \%$. 白色固体, m.p. $135 \sim 136{ }^{\circ} \mathrm{C} ;{ }^{1} \mathrm{H}$ NMR $(600$ $\left.\mathrm{MHz}, \mathrm{CDCl}_{3}\right) \delta: 7.81(\mathrm{~s}, 1 \mathrm{H}), 7.66 \sim 7.61(\mathrm{~m}, 4 \mathrm{H}), 5.66(\mathrm{t}$, $J=5.4 \mathrm{~Hz}, 1 \mathrm{H}), 4.65 \sim 4.61(\mathrm{~m}, 1 \mathrm{H}) ;{ }^{13} \mathrm{C}$ NMR $(150 \mathrm{MHz}$, $\left.\mathrm{CDCl}_{3}\right) \delta: 167.9,132.5,129.8,128.9,128.2,121.5$ (q, $J=$ $279.9 \mathrm{~Hz}), 111.4,54.9$ (q, $J=33.3 \mathrm{~Hz}) ;{ }^{19} \mathrm{~F}$ NMR $(376$ $\left.\mathrm{MHz}, \mathrm{CDCl}_{3}\right) \delta:-73.30(\mathrm{~d}, J=6.4 \mathrm{~Hz})$; IR (KBr) $v$ : 3428, 3313, 2964, 1648, 1539, 1513, 1474, 1261, 1203, $1153 \mathrm{~cm}^{-1}$; HRMS (ESI) calcd for $\mathrm{C}_{10} \mathrm{H}_{7} \mathrm{BrF}_{3} \mathrm{~N}_{3} \mathrm{ONa}[\mathrm{M}+$ $\mathrm{Na}]^{+}$343.9617, found 343.9608.

$N^{\prime}$-(1,1,1-三氟丙腈-2-基)-4-氟苯甲酰肼 $(3 \mathbf{j}): 24 \mathrm{mg}$, 产率 92\%. 白色固体, m.p. $113 \sim 114{ }^{\circ} \mathrm{C} ;{ }^{1} \mathrm{H}$ NMR $(600$ $\left.\mathrm{MHz}, \mathrm{CDCl}_{3}\right) \delta: 7.85(\mathrm{~s}, 1 \mathrm{H}), 7.83 \sim 7.80(\mathrm{~m}, 2 \mathrm{H}), 7.16(\mathrm{t}$, $J=9.0 \mathrm{~Hz}, 2 \mathrm{H}), 5.71(\mathrm{t}, J=4.8 \mathrm{~Hz}, 1 \mathrm{H}), 4.67 \sim 4.63(\mathrm{~m}$, $1 \mathrm{H}) ;{ }^{13} \mathrm{C}$ NMR $\left(150 \mathrm{MHz}, \mathrm{CDCl}_{3}\right) \delta: 167.8,165.7(\mathrm{~d}, J=$ $253.2 \mathrm{~Hz}), 129.9(\mathrm{~d}, J=9.2 \mathrm{~Hz}), 127.2(\mathrm{~d}, J=3.3 \mathrm{~Hz})$, 121.6 (q, $J=280.4 \mathrm{~Hz}), 116.4$ (d, $J=22.1 \mathrm{~Hz}), 111.5,54.9$ (q, $J=33.3 \mathrm{~Hz}) ;{ }^{19} \mathrm{~F}$ NMR $\left(376 \mathrm{MHz}, \mathrm{CDCl}_{3}\right) \delta:-73.28$ (d, $J=6.4 \mathrm{~Hz}),-105.44 \sim-105.51(\mathrm{~m})$; IR (KBr) $v$ : $3441,3273,2957,1650,1605,1531,1514,1472,1323$, 1256, 1212, $1154 \mathrm{~cm}^{-1}$; HRMS (ESI) calcd for $\mathrm{C}_{10} \mathrm{H}_{7} \mathrm{~F}_{4^{-}}$ $\mathrm{N}_{3} \mathrm{ONa}[\mathrm{M}+\mathrm{Na}]^{+}$284.0417, found 284.0411.

$N^{\prime}$-(1,1,1-三氟丙腈-2-基)-4-三氟甲基苯甲酰肼 $(\mathbf{3 k}$ ): $26 \mathrm{mg}$, 产率 $84 \%$. 白色固体, m.p. $150 \sim 151{ }^{\circ} \mathrm{C} ;{ }^{1} \mathrm{H}$
NMR $\left(600 \mathrm{MHz}, \mathrm{CDCl}_{3}\right) \delta: 7.92(\mathrm{~d}, J=8.4 \mathrm{~Hz}, 2 \mathrm{H})$, $7.78 \sim 7.76(\mathrm{~m}, 3 \mathrm{H}), 5.62(\mathrm{t}, J=4.8 \mathrm{~Hz}, 1 \mathrm{H}), 4.66 \sim 4.62$ $(\mathrm{m}, 1 \mathrm{H}) ;{ }^{13} \mathrm{C}$ NMR $\left(150 \mathrm{MHz}, \mathrm{CD}_{3} \mathrm{OD}\right) \delta: 169.3,137.3$, 134.6 (q, $J=32.4 \mathrm{~Hz}), 129.4,126.6$ (q, $J=3.9 \mathrm{~Hz}), 125.2$ (q, $J=270.2 \mathrm{~Hz}), 123.6$ (q, $J=278.7 \mathrm{~Hz}), 113.6,55.3$ (q, $J=32.7 \mathrm{~Hz}) ;{ }^{19} \mathrm{~F} \mathrm{NMR}\left(376 \mathrm{MHz}, \mathrm{CDCl}_{3}\right) \delta:-63.61(\mathrm{~s})$, -73.27 (d, $J=6.0 \mathrm{~Hz}$ ); IR (KBr) $v: 3441,2928,1650$, 1538, 1514, 1460, 1331, 1261, 1215, $1133 \mathrm{~cm}^{-1}$; HRMS (ESI) calcd for $\mathrm{C}_{11} \mathrm{H}_{7} \mathrm{~F}_{6} \mathrm{~N}_{3} \mathrm{ONa}[\mathrm{M}+\mathrm{Na}]^{+} 334.0386$, found 334.0371 .

$N^{\prime}$-(1,1,1-三氟丙腈-2-基)-2-萗甲酰肼(3I): $24 \mathrm{mg}$, 产 率 $82 \%$. 白色固体, m.p. $154 \sim 155{ }^{\circ} \mathrm{C} ;{ }^{1} \mathrm{H}$ NMR $(600$ $\left.\mathrm{MHz}, \mathrm{CDCl}_{3}\right) \delta: 8.33(\mathrm{~s}, 1 \mathrm{H}), 7.95 \sim 7.92(\mathrm{~m}, 3 \mathrm{H}), 7.89(\mathrm{~d}$, $J=8.4 \mathrm{~Hz}, 1 \mathrm{H}), 7.81(\mathrm{~d}, J=8.4 \mathrm{~Hz}, 1 \mathrm{H}), 7.61$ (t, $J=6.6$ $\mathrm{Hz}, 1 \mathrm{H}), 7.57$ (t, $J=7.2 \mathrm{~Hz}, 1 \mathrm{H}), 5.71(\mathrm{t}, J=5.4 \mathrm{~Hz}, 1 \mathrm{H})$, $4.71 \sim 4.67(\mathrm{~m}, 1 \mathrm{H}) ;{ }^{13} \mathrm{C}$ NMR $\left(150 \mathrm{MHz}, \mathrm{CDCl}_{3}\right) \delta$ : $168.9,135.5,132.6,129.2,129.1,128.6,128.5,128.1$, $128.0,127.4,123.2,121.6(\mathrm{q}, J=280.1 \mathrm{~Hz}), 111.6,55.0$ (q, $J=33.0 \mathrm{~Hz}) ;{ }^{19} \mathrm{~F}$ NMR $\left(376 \mathrm{MHz}, \mathrm{CDCl}_{3}\right) \delta:-61.08(\mathrm{~d}$, $J=6.4 \mathrm{~Hz}$ ); IR (KBr) $v: 3431,2960,1650,1516,1474$, $1355,1309,1264,1199,1151 \mathrm{~cm}^{-1}$; HRMS (ESI) calcd for $\mathrm{C}_{14} \mathrm{H}_{10} \mathrm{~F}_{3} \mathrm{~N}_{3} \mathrm{ONa}[\mathrm{M}+\mathrm{Na}]^{+} 316.0668$, found 316.0653 .

$N^{\prime}$-(1,1,1-三氟丙腈-2-基)-2-呋喃甲酰肼(3m): 14 $\mathrm{mg}$, 产率 60\%. 白色固体, m.p. $126 \sim 127{ }^{\circ} \mathrm{C} ;{ }^{1} \mathrm{H}$ NMR $\left(600 \mathrm{MHz}, \mathrm{CDCl}_{3}\right) \delta: 7.97(\mathrm{~s}, 1 \mathrm{H}), 7.52 \sim 7.51(\mathrm{~m}, 1 \mathrm{H})$, $7.26(\mathrm{~s}, 1 \mathrm{H}), 6.57$ (q, $J=1.8 \mathrm{~Hz}, 1 \mathrm{H}), 5.57$ (t, $J=4.8 \mathrm{~Hz}$, $1 \mathrm{H}), 4.66 \sim 4.62(\mathrm{~m}, 1 \mathrm{H}) ;{ }^{13} \mathrm{C}$ NMR $\left(150 \mathrm{MHz}, \mathrm{CDCl}_{3}\right) \delta$ : 159.1, 145.4, 145.3, 121.5 (q, $J=280.2 \mathrm{~Hz}$ ), 117.1, 112.7, $111.5,55.0$ (q, $J=33.6 \mathrm{~Hz}) ;{ }^{19} \mathrm{~F}$ NMR $\left(376 \mathrm{MHz}, \mathrm{CDCl}_{3}\right.$ ) $\delta:-73.35$ (d, $J=6.4 \mathrm{~Hz})$; IR (KBr) v: 3439, 1649, 1516, 1309, 1259, 1199, $1149 \mathrm{~cm}^{-1}$; HRMS (ESI) calcd for $\mathrm{C}_{8} \mathrm{H}_{6} \mathrm{~F}_{3} \mathrm{~N}_{3} \mathrm{O}_{2} \mathrm{Na}[\mathrm{M}+\mathrm{Na}]^{+}$256.0304, found 256.0292.

$N^{\prime}$-(1,1,1-三氟丙腈-2-基)-异丁酰肼(3n): $14 \mathrm{mg}$, 产 率 67\%. 白色固体, m.p. $106 \sim 108{ }^{\circ} \mathrm{C} ;{ }^{1} \mathrm{H}$ NMR (600 $\left.\mathrm{MHz}, \mathrm{CDCl}_{3}\right) \delta: 7.17(\mathrm{~s}, 1 \mathrm{H}), 5.37(\mathrm{t}, J=5.4 \mathrm{~Hz}, 1 \mathrm{H})$, $4.56 \sim 4.52(\mathrm{~m}, 1 \mathrm{H}), 2.46 \sim 2.39(\mathrm{~m}, 1 \mathrm{H}), 1.23(\mathrm{~d}, J=6.6$ $\mathrm{Hz}, 3 \mathrm{H}), 1.20(\mathrm{~d}, J=7.2 \mathrm{~Hz}, 3 \mathrm{H}) ;{ }^{13} \mathrm{C}$ NMR $(150 \mathrm{MHz}$, $\left.\mathrm{CDCl}_{3}\right) \delta: 178.0,121.6(\mathrm{q}, J=280.2 \mathrm{~Hz}), 111.2,54.8(\mathrm{q}$, $J=33.0 \mathrm{~Hz}), 33.7,19.5,19.1 ;{ }^{19} \mathrm{~F}$ NMR $(376 \mathrm{MHz}$, $\left.\mathrm{CDCl}_{3}\right) \delta:-73.48(\mathrm{~d}, J=6.4 \mathrm{~Hz})$; IR (KBr) v: 3441, 2978, 2934, 1677, 1264, 1196, $1151 \mathrm{~cm}^{-1}$; HRMS (ESI) calcd for $\mathrm{C}_{7} \mathrm{H}_{10} \mathrm{~F}_{3} \mathrm{~N}_{3} \mathrm{ONa}[\mathrm{M}+\mathrm{Na}]^{+} 232.0668$, found 232.0660 .

$N^{\prime}$-(1,1,1-三氟丙腈-2-基)-特戊酰肼(3o): $17 \mathrm{mg}$, 产 率 76\%. 白色固体, m.p. $153 \sim 154{ }^{\circ} \mathrm{C} ;{ }^{1} \mathrm{H}$ NMR $(600$ 
$\left.\mathrm{MHz}, \mathrm{CDCl}_{3}\right) \delta: 7.33(\mathrm{~s}, 1 \mathrm{H}), 5.42(\mathrm{~d}, J=4.8 \mathrm{~Hz}, 1 \mathrm{H})$, $4.56 \sim 4.52(\mathrm{~m}, 1 \mathrm{H}), 1.26(\mathrm{~s}, 9 \mathrm{H}) ;{ }^{13} \mathrm{C}$ NMR $(150 \mathrm{MHz}$, $\left.\mathrm{CDCl}_{3}\right) \delta: 179.5,121.6(\mathrm{q}, J=279.9 \mathrm{~Hz}), 111.2,54.8(\mathrm{q}$, $J=33.0 \mathrm{~Hz}), 38.3,27.2 ;{ }^{19} \mathrm{~F}$ NMR $\left(376 \mathrm{MHz}, \mathrm{CDCl}_{3}\right) \delta$ : -73.48 (d, $J=6.4 \mathrm{~Hz}$ ); IR (KBr) $v: 3368,2969,1662$, $1524,1192,1154 \mathrm{~cm}^{-1}$; HRMS (ESI) calcd for $\mathrm{C}_{8} \mathrm{H}_{12} \mathrm{~F}_{3}$ $\mathrm{N}_{3} \mathrm{ONa}[\mathrm{M}+\mathrm{Na}]^{+} 246.0825$, found 246.0831 .

$N^{\prime}$-(1,1,1-三氟丙腈-2-基)-环己基甲酰肼(3p): $18 \mathrm{mg}$, 产率 72\%. 白色固体, m.p. $138 \sim 140{ }^{\circ} \mathrm{C} ;{ }^{1} \mathrm{H}$ NMR (600 $\left.\mathrm{MHz}, \mathrm{CDCl}_{3}\right) \delta: 7.17(\mathrm{~s}, 1 \mathrm{H}), 5.50 \sim 5.38(\mathrm{~m}, 1 \mathrm{H}), 4.58 \sim$ $4.52(\mathrm{~m}, 1 \mathrm{H}), 2.19 \sim 2.14(\mathrm{~m}, 1 \mathrm{H}), 1.91 \sim 1.81(\mathrm{~m}, 4 \mathrm{H})$, $1.70 \sim 1.67(\mathrm{~m}, 1 \mathrm{H}), 1.53 \sim 1.46(\mathrm{~m}, 2 \mathrm{H}), 1.30 \sim 1.21(\mathrm{~m}$, $3 \mathrm{H}) ;{ }^{13} \mathrm{C}$ NMR $\left(150 \mathrm{MHz}, \mathrm{CDCl}_{3}\right) \delta: 177.0,121.6$ (q, $J=$ $280.4 \mathrm{~Hz}), 111.3,54.8$ (q, $J=33.0 \mathrm{~Hz}), 43.4,29.5,29.2$, 25.6, 25.6, 25.5; ${ }^{19} \mathrm{~F}$ NMR $\left(376 \mathrm{MHz}, \mathrm{CDCl}_{3}\right) \delta:-73.46$ (d, $J=6.4 \mathrm{~Hz}$ ); IR (KBr) $v: 3441,3357,2938,1678,1262$, 1196, $1150 \mathrm{~cm}^{-1}$; HRMS (ESI) calcd for $\mathrm{C}_{10} \mathrm{H}_{14} \mathrm{~F}_{3} \mathrm{~N}_{3} \mathrm{ONa}$ $[\mathrm{M}+\mathrm{Na}]^{+}$272.0981, found 272.0970.

$N^{r}$-(1,1,1-三氟丙腈-2-基)-苯乙酰肼(3q): $20 \mathrm{mg}$, 产 率 78\%. 白色固体, m.p. 84 86 ${ }^{\circ} \mathrm{C} ;{ }^{1} \mathrm{H}$ NMR $(600 \mathrm{MHz}$, $\left.\mathrm{CDCl}_{3}\right) \delta: 7.38(\mathrm{t}, J=7.2 \mathrm{~Hz}, 2 \mathrm{H}), 7.33(\mathrm{t}, J=7.2 \mathrm{~Hz}, 1 \mathrm{H})$, 7.27 (d, $J=7.2 \mathrm{~Hz}, 2 \mathrm{H}), 7.14$ (s, 1H), 5.44 (d, $J=5.4 \mathrm{~Hz}$, $1 \mathrm{H}), 4.52 \sim 4.48(\mathrm{~m}, 1 \mathrm{H}), 3.66(\mathrm{~d}, J=16.2 \mathrm{~Hz}, 1 \mathrm{H}), 3.63$ $(\mathrm{d}, J=16.2 \mathrm{~Hz}, 1 \mathrm{H}) ;{ }^{13} \mathrm{C}$ NMR $\left(150 \mathrm{MHz}, \mathrm{CDCl}_{3}\right) \delta$ : $172.0,132.9,129.5,129.4,128.0,121.5(\mathrm{q}, J=280.1 \mathrm{~Hz})$, $111.5,54.6$ (q, $J=33.3 \mathrm{~Hz}), 41.6 ;{ }^{19} \mathrm{~F}$ NMR $(376 \mathrm{MHz}$, $\left.\mathrm{CDCl}_{3}\right) \delta:-73.48(\mathrm{~d}, J=6.4 \mathrm{~Hz})$; IR (KBr) v: 3439, 2941, 1653, 1260, 1201, $1151 \mathrm{~cm}^{-1}$; HRMS (ESI) calcd for $\mathrm{C}_{11} \mathrm{H}_{10} \mathrm{~F}_{3} \mathrm{~N}_{3} \mathrm{ONa}[\mathrm{M}+\mathrm{Na}]^{+} 280.0668$, found 280.0665 .

$N^{\prime}$-(1,1,1-三氟丙腈-2-基)-月桂酰胇(3r): $29 \mathrm{mg}$, 产 率 90\%. 白色固体, m.p. $77 \sim 78{ }^{\circ} \mathrm{C} ;{ }^{1} \mathrm{H}$ NMR $(600 \mathrm{MHz}$, $\left.\mathrm{CDCl}_{3}\right) \delta: 7.23(\mathrm{~s}, 1 \mathrm{H}), 5.45(\mathrm{t}, J=4.8 \mathrm{~Hz}, 1 \mathrm{H}), 4.57 \sim 4.53$ $(\mathrm{m}, 1 \mathrm{H}), 2.28 \sim 2.20(\mathrm{~m}, 2 \mathrm{H}), 1.69 \sim 1.64(\mathrm{~m}, 2 \mathrm{H}), 1.34 \sim$ $1.26(\mathrm{~m}, 16 \mathrm{H}), 0.88(\mathrm{~d}, J=7.2 \mathrm{~Hz}, 3 \mathrm{H}) ;{ }^{13} \mathrm{C}$ NMR $(150$ $\left.\mathrm{MHz}, \mathrm{CDCl}_{3}\right) \delta: 174.3,121.5$ (q, $\left.J=280.1 \mathrm{~Hz}\right), 111.5,54.8$ (q, $J=33.0 \mathrm{~Hz}$ ), 34.3, 32.0, 29.7, 29.6, 29.5, 29.4, 29.3, 25.4, 22.8, 14.2; ${ }^{19} \mathrm{~F}$ NMR $\left(376 \mathrm{MHz}, \mathrm{CDCl}_{3}\right) \delta:-73.48$ (d, $J=4.1 \mathrm{~Hz})$; IR (KBr) $v$ : 3441, 2925, 1647, 1260, 1197, $1151 \mathrm{~cm}^{-1}$; HRMS (ESI) calcd for $\mathrm{C}_{15} \mathrm{H}_{26} \mathrm{~F}_{3} \mathrm{~N}_{3} \mathrm{ONa}[\mathrm{M}+$ $\mathrm{Na}]^{+} 344.1920$, found 344.1905.

\section{2 .2 化合物 7 的合成}

在 $50 \mathrm{~mL}$ 的圆底烧瓶中, 依次加入 $N^{\prime}-(1,1,1$-三氟丙 腈-2-基)苯甲酰肼(3a) $(50 \mathrm{mg}, 0.2 \mathrm{mmol})$ 和 $3 \mathrm{~mL}$ 甲醇, 在 $0{ }^{\circ} \mathrm{C}$ 下滴加盐酸甲醇(将氯化氢气体通入无水甲醇中) 溶液 $0.4 \mathrm{~mL}$, 将此混合液在摚拌下回流. 薄层色谱
(TLC)检测反应，待反应完全后除去溶剂. 然后，向剩余 反应液中加入 $6 \mathrm{~mL}$ 饱和碳酸氢钠溶液, 搅拌 $10 \mathrm{~min}$, 用乙酸乙酯萃取 $(10 \mathrm{~mL} \times 3)$. 合并有机相, 用无水硫酸 镁干燥, 蒸去溶剂后, 进行柱层析 $[V$ (石油醚) $: V($ 乙酸 乙酯) $=4: 1$ ]分离得产物 $N^{\prime}-(1,1,1$-三氟丙酸甲酯-2-基) 苯甲酰肼(7a) $36 \mathrm{mg}$, 产率 64\%. 白色固体, m.p. 88 $90{ }^{\circ} \mathrm{C} ;{ }^{1} \mathrm{H}$ NMR $\left(400 \mathrm{MHz}, \mathrm{CDCl}_{3}\right) \delta: 8.06(\mathrm{~s}, 1 \mathrm{H}), 7.76$ (d, $J=7.2 \mathrm{~Hz}, 2 \mathrm{H}), 7.55$ (t, $J=6.0 \mathrm{~Hz}, 1 \mathrm{H}), 7.45$ (t, $J=7.2$ $\mathrm{Hz}, 2 \mathrm{H}), 5.56(\mathrm{~s}, 1 \mathrm{H}), 4.22 \sim 4.16(\mathrm{~m}, 1 \mathrm{H}), 3.89(\mathrm{~s}, 3 \mathrm{H})$; ${ }^{13} \mathrm{C} \mathrm{NMR}\left(150 \mathrm{MHz}, \mathrm{CDCl}_{3}\right) \delta: 168.2,165.6,132.6,131.8$, $128.9,127.1,123.3(\mathrm{q}, J=279.9 \mathrm{~Hz}), 65.0(\mathrm{q}, J=29.1 \mathrm{~Hz})$, 53.7; ${ }^{19} \mathrm{~F}$ NMR $\left(376 \mathrm{MHz}, \mathrm{CDCl}_{3}\right) \delta:-72.72(\mathrm{~d}, J=7.5$ Hz); IR (KBr) v: 3290, 2971, 2901, 2272, 1752, 1651, 1522, 1473, 1265, 1224, $1120 \mathrm{~cm}^{-1}$; HRMS (ESI) calcd for $\mathrm{C}_{11} \mathrm{H}_{11} \mathrm{~F}_{3} \mathrm{~N}_{2} \mathrm{O}_{3} \mathrm{Na}[\mathrm{M}+\mathrm{Na}]^{+}$299.0614, found 299.0612 .

\section{化合物 7b 的合成方法同 7a.}

$N^{\prime}$-(1,1,1-三氟丙酸甲酯-2-基)-2-甲基苯甲酰肼(7b): $30 \mathrm{mg}$, 产率 52\%. 无色油状液体. ${ }^{1} \mathrm{H}$ NMR $(400 \mathrm{MHz}$, $\left.\mathrm{CDCl}_{3}\right) \delta: 7.52(\mathrm{~s}, 1 \mathrm{H}), 7.43 \sim 7.32(\mathrm{~m}, 2 \mathrm{H}), 7.28 \sim 7.20$ (m, 2H), $5.54(\mathrm{~s}, 1 \mathrm{H}), 4.24 \sim 4.17(\mathrm{~m}, 1 \mathrm{H}), 3.91(\mathrm{~s}, 3 \mathrm{H})$, 2.43 (s, $3 \mathrm{H}) ;{ }^{13} \mathrm{C}$ NMR $\left(150 \mathrm{MHz}, \mathrm{CDCl}_{3}\right) \delta: 170.2,165.4$, $137.2,132.9,131.5,131.1,127.2,126.0,123.3$ (q, $J=$ $279.8 \mathrm{~Hz}), 64.9$ (q, $J=29.4 \mathrm{~Hz}), 53.7,19.9 ;{ }^{19} \mathrm{~F}$ NMR (376 $\left.\mathrm{MHz}, \mathrm{CDCl}_{3}\right) \delta:-72.62(\mathrm{~d}, J=7.5 \mathrm{~Hz})$; IR $(\mathrm{KBr}) v$ : $3277,3033,2955,1751,1652,1514,1458,1314,1264$, $1223,1184,1127 \mathrm{~cm}^{-1}$; HRMS (ESI) calcd for $\mathrm{C}_{12} \mathrm{H}_{13} \mathrm{~F}_{3}-$ $\mathrm{N}_{2} \mathrm{O}_{3} \mathrm{Na}[\mathrm{M}+\mathrm{Na}]^{+}$313.0770, found 313.0775.

辅助材料(Supporting Information) 合成产物的 ${ }^{1} \mathrm{H}$ $\mathrm{NMR},{ }^{13} \mathrm{C} \mathrm{NMR}$ 和 ${ }^{19} \mathrm{~F}$ NMR 以及高分辨质谱谱图. 这些 材料可以免费从本刊网站(http://sioc-journal.cn/)上下载.

\section{References}

[1] (a) Chauhan, J.; Ravva, M. K.; Sen, S. Org. Lett. 2019, 21, 6562. (b) Wang, Y.; Wang, K.-H.; Su, Y.; Yang, Z.; Wen, L.; Liu, L.; Wang, J.; Huang, D.; Hu, Y. J. Org. Chem. 2018, 83, 939.

(c) Tokumaru, K.; Johnston, J. N. Chem. Sci. 2017, 8, 3187.

(d) An, J.; Alper, H.; Beauchemin, A. M. Org. Lett. 2016, 18, 3482.

(e) Fan, Y.; He, Y.; Liu, X.; Hu, T.; Ma, H.; Yang, X.; Luo, X.; Huang, G. J. Org. Chem. 2016, 81, 6820.

(f) Flagstad, T.; Petersen, M. T.; Nielsen, T. E. Angew. Chem., Int. Ed. 2015, 54, 8395 .

[2] (a) Yoneya, M.; Takada, S.; Maeda, Y.; Yokoyama, H. Liq. Cryst. 2008, 35, 339.

(b) Parra, M.; Hidalgo, P.; Barberá, J.; Carrasco, E.; Saavedra, C. Liq. Cryst. 2006, 33, 391.

(c) Cui, H.; Xu, Y.; Zhang, Z.-F. Anal. Chem. 2004, 76, 4002.

(d) Zhao, X.; Wang, X.-Z.; Jiang, X.-K.; Chen, Y.-Q.; Li, Z.-T.; Chen, G.-J. J. Am. Chem. Soc. 2003, 125, 15128.

[3] Wing, K. D. Science 1988, 241, 467. 
[4] (a) Wheelock, C. E.; Nakagawa, Y.; Harada, T.; Oikawa, N.; Akamatsu, M.; Smagghe, G.; Stefanou, D.; Iatrou, K.; Swevers, L. Bioorg. Med. Chem. 2006, 14, 1143.

(b) Nakagawa, Y. Vitam. Horm. 2005, 73, 131.

(c) Wing, K. D.; Slawecki, R. A.; Carlson, G. R. Science 1988, 241, 470 .

[5] (a) Toraskar, M. P.; Kadam, V. J.; Kulkarni, V. M. Int. J. ChemTech Res. 2009, 1, 1194.

(b) Lian, S.; Su, H.; Zhao, B.-X.; Liu, W.-Y.; Zheng, L.-W.; Miao, J.-Y. Bioorg. Med. Chem. 2009, 17, 7085.

(c) Duarte, C. D.; Tributino, J. L. M.; Lacerda, D. I.; Martins, M. V.; Alexandre-Moreira, M. S.; Dutra, F.; Bechara, E. J. H.; De-Paula, F. S.; Goulart, M. O. F.; Ferreira, J.; Calixto, J. B.; Nunes, M. P.; Bertho, A. L.; Miranda, A. L. P.; Barreiro, E. J.; Fraga, C. A. M. Bioorg. Med. Chem. 2007, 15, 2421.

[6] (a) Formicola, L.; Maréchal, X.; Basse, N.; Bouvier-Durand, M.; Bonnet-Delpon, D.; Milcent, T.; Reboud-Ravaux, M.; Ongeri, S. Bioorg. Med. Chem. Lett. 2009, 19, 83.

(b) Onnis, V.; Cocco, M. T.; Fadda, R.; Congiu, C. Bioorg. Med. Chem. 2009, 17, 6158.

[7] (a) Schneider, U.; Chen, I.-H; Kobayashi, S. Org. Lett. 2008, 10, 737.

(b) Tan, K. L.; Jacobsen, E. N. Angew. Chem., Int. Ed. 2007, 46, 1315 .

(c) Sugiura, M.; Kobayashi, S. Angew. Chem., Int. Ed. 2005, 44, 5176 .

(d) Hirabayashi, R.; Ogawa, C.; Sugiura, M.; Kobayashi, S. J. Am. Chem. Soc. 2001, 123, 9493.

[8] (a) Alonso, C.; de Marigorta, E. M.; Rubiales, G.; Palacios, F. Chem. Rev. 2015, 115, 1847.

(b) Wang, J.; Sánchez-Roselló, M.; Aceña, J. L.; Pozo, C.; Sorochinsky, A. E.; Fustero, S.; Soloshonok, V. A.; Liu, H. Chem. Rev. 2014, 114, 2432.

(c) Liang, T.; Neumann, C. N.; Ritter, T. Angew. Chem., Int. Ed. 2013, 52, 8214 .

(d) Qing, F. Chin. J. Org. Chem. 2012, 32, 815 (in Chinese).

(卿凤翎, 有机化学, 2012, 32, 815.)

(e) Hagmann, W. K. J. Med. Chem. 2008, 51, 4359.

(f) Müller, K.; Faeh, C.; Diederich, F. Science 2007, 317, 1881.

[9] For selected reviews, see:

(a) Moschner, J.; Stulberg, V.; Fernandes, R.; Huhmann, S.; Leppkes, J.; Koksch, B. Chem. Rev. 2019, 119, 10718.

(b) Fustero, S.; Simón-Fuentes, A.; Barrio, P.; Haufe, G. Chem. Rev. 2015, 115,871 .

(c) Nie, J.; Guo, H.-C.; Cahard, D.; Ma, J.-A. Chem. Rev. 2011, 111,
455.

(d) Uneyama, K.; Katagiri, T.; Amii, H. Acc. Chem. Res. 2008, 41, 817.

(e) Schlosser, M. Angew. Chem., Int. Ed. 2006, 45, 5432.

[10] For selected examples, see:

(a) Yang, Y.-Y.; Yang, G.; Cheng, C.; Li, Y.-X.; Zhang, J.-Q.; Feng, W.; Zhao, Y.-L.; Tang, L. Org. Lett. 2019, 21, 2236.

(b) Liu, J.; Huang, D.; Wang, X.; Zong, W.; Su, Y.; Wang, K.-H.; Hu, Y. Chin. J. Org. Chem. 2019, 39, 1767 (in Chinese). (刘佳欣，黄丹凤，王小平，宗吴中，苏瀛鹏，王克虎，胡雨来， 有机化学, 2019, 39, 1767.)

(c) You, Y.; Lu, W.-Y.; Wang, Z.-H.; Chen, Y.-Z.; Xu, X.-Y.; Zhang, X.-M.; Yuan, W.-C. Org. Lett. 2018, 20, 4453.

(d) Wang, J.; Li, F.; Xu, Y.; Wang, J.; Wu, Z.; Yang, C.; Liu, L. Chin. J. Org. Chem. 2018, 38, 1155 (in Chinese).

(王晶晶, 李峰, 徐妍, 王娟, 武紫燕, 杨成玉, 刘澜涛, 有机化 学, 2018, 38, 1155.)

(e) Zhang, F.-G.; Lv, N.; Zheng, Y.; Ma, J.-A. Chin. J. Chem. 2018, 36, 723 .

[11] (a) Li, J.; Yang, T.; Zhang, H.; Huang, D.; Wang, K.-H.; Su, Y.; Hu, Y. Chin. J. Org. Chem. 2017, 37, 925 (in Chinese). (李军, 杨天宇, 张怀远, 黄丹凤, 王克虎, 苏瀛鹏, 胡雨来, 有 机化学, 2017, 37, 925.)

(b) Li, J.; Huang, D.; Zhang, H.; Zhang, X.; Wang, J.; Wang, K.-H.; Su, Y.; Hu, Y. Chin. J. Org. Chem. 2017, 37, 2985 (in Chinese). (李军, 黄丹凤, 张怀远, 张兴虎, 王娟娟, 王克虎, 苏瀛鹏, 胡 雨来, 有机化学, 2017, 37, 2985.)

(c) Peng, X.; Wang, K.-H.; Huang, D.; Wang, J.; Wang, Y.; Su, Y.; Hu, Y.; Fu, Y. Appl. Organomet. Chem. 2017, 31, 3731.

(d) Du, G.; Huang, D.; Wang, K.-H.; Chen, X.; Xu, Y.; Ma, J.; Su, Y.; Fu, Y.; Hu, Y. Org. Biomol. Chem. 2016, 14, 1492.

[12] (a) Wen, L.; Huang, D.; Wang, K.-H.; Wang, Y.; Liu, L.; Yang, Z.; $\mathrm{Su}, \mathrm{Y}$; Hu, Y. Synthesis 2018, 50, 1979.

(b) Wang, K.-H.; Wang, J.; Wang, Y.; Su, Y.; Huang, D.; Fu, Y.; Du, Z.; Hu, Y. Synthesis 2018, 50, 1907.

(c) Liu, L.; Huang, D.; Wang, Y.; Wen, L.; Yang, Z; Su, Y.; Wang, K.-H.; Hu, Y. Chin. J. Org. Chem. 2018, 38, 1469 (in Chinese).

(刘丽丽, 黄丹凤, 王玉祥, 文岗, 杨政, 苏瀛鹏, 王克虎, 胡雨 来, 有机化学, 2018, 38, 1469.)

(d) Peng, X.; Huang, D.; Wang, K.-H.; Wang, Y.; Wang, J.; Su, Y.; Hu, Y. Org. Biomol. Chem. 2017, 15, 6214

[13] (a) Zamfir, A.; Tsogoeva, S. B. Org. Lett. 2010, 12, 188.

(b) Keith, J. M.; Jacobsen, E. N. Org. Lett. 2004, 6, 153.

(c) Ding, H.; Friestad, G. K. Heterocycles 2006, 70, 185. 